\title{
Electrodynamic linear response of a superconductor film located at the surface of semiconductor
}

\author{
V. Lozovski, D. Reznik \\ V. Lashkaryov Institute of Semiconductor Physics, NAS of Ukraine, 41, pr. Nauky, 03028 Kyiv, Ukraine \\ E-mail:vlozovski@isp.kiev.ua
}

\begin{abstract}
The susceptibility of thin superconductor film was obtained. The absorption of energy of external electromagnetic field by superconductor film located at a semiconductor substrate was calculated. A new approach to calculation of the dispersion relation for eigenmodes of a strong inhomogeneous system was developed. The dispersion relations of eigenmodes of a superconductor thin film on the semiconductor substrate was calculated using the developed approach.
\end{abstract}

Keywords: linear response, superconductivity, local field, surface, energy absorption.

Paper received 25.11.03; accepted for publication 30.03.04.

\section{Introduction}

The superconductor-semiconductor systems are actual because the extremely low level of self-noises is the main characteristic of the systems. In this connection the electrodynamics of thin superconductor film at semiconductor substrate is the actual problem. The study of superconductor-semiconductor systems is of interest now. Numerous works are devoted to consideration of different features in behavior of the superconductor-semiconductor systems. The works touch upon both fundamental and application aspects of the problem as well. In [1] the thin superconducting $\mathrm{LaSrCuO}$ film electrical and optical properties were studied. In [2] presented were some superconductor-semiconductor devices. It was, for example, the superconductor straight-line resonator that possesses has an electromagnetic feed-through level below $-65 \mathrm{~dB}$ up to $10 \mathrm{GHz}$. In this work, a semiconductorsuperconductor microwave digital phase shifter based on an $\mathrm{YbaCuO}$ thin film was demonstrated. The organic layered superconductor composites are synthesized and studied (see, for example [3]). The electrodynamical properties of various superconducting structures are intensively studied, too. Thus, in Ref.[4] the measurements of the emitted spectra for electromagnetic waves of the teraHertz range from tunnel-injected non-equilibrium $\mathrm{YbaCuO}$ superconductor were carried out. The observed spectra have a single broad-picked structure with the peak frequency about $5 \mathrm{THz}$. The linear and nonlinear electromagnetic properties of superconducting systems are widely studied (see, for example [5]). All these studies suggest that the study of interaction between external electromagnetic field and the system containing superconductor nano-systems (including thin films) is very important and actual problem.

The characteristic dispersion properties for superconductors obviously are in the range of frequencies close to superconducting energy gap, because the state density of superconductor quasi-particles is rather high just near the energy gap. For the most "classic" superconductors these frequencies are small $\left(10^{11}-10^{12} \mathrm{~Hz}\right)$ as comparable to characteristic frequencies of semiconductor (the optical phonons frequencies, for example). One can think that more interesting could be the systems consisting of high- $T_{C}$ (HTSC) structures at a semiconductor substrate. These systems are characterized by the gap frequencies about $10^{13}-10^{14} \mathrm{~Hz}$ that lie in the range of characteristic optical phonon frequencies of semiconductors. As a result, for superconducting thin film with the thickness $h \approx 100 \mathrm{~nm}$ being comparable with the Pippard penetration depth, the wavelength of electromagnetic waves corresponding to the resonant range of the frequencies are much larger than $h$. Then, the near-field approximation can be used in the studies of these systems. On the other hand, at so small distances the local field are rapidly varied and these inhomogeneities have to be taken into 


\section{Lozovski, D. Reznik: Electrodynamic linear response of a superconductor film ...}

account. Then the non-local electrodynamical interactions inside the system can result in arising the local oscillations. These oscillation processes are caused by interaction between electromagnetic field and local currents inside the system. The local currents depend on electron and phonon excitations. Then, the oscillating electromagnetic field is characterized by the frequencies dependent on the electronic properties of the system as well as the shape and dimension of superconducting system. The generalized excitations that arise in the system are eigenmodes of the system. These excitations characterize the system as a whole. The dispersion relation of these oscillations - the connection between the resonant frequencies and wave vector of the external field that causes the oscillations - are the important characteristic of the system. The dispersion relations define the response of the system on the external electromagnetic field and are the most important characteristic.

This work is devoted to calculations of the non-local effective susceptibility of thin superconductor film at the semiconductor surface. The calculations are made in the frame of the near-field approximation [6] in the mesoscopic approach. Unlike most of works where the electrodynamical properties of thin films are considered (see, for example [7]), the developed approach is based on the solution of the Lippmann-Schwinger equation for selfconsistent field [8], which enabled us to calculate the effective susceptibility directly. The main idea of determination of the dispersion relations in the inhomogeneous system is the calculation of the absorbed energy by the system of external electromagnetic field, because this absorption have to extremely increase when frequency and wave vector of external field correspond to eigenmodes of the system.

\section{Dispersion relations}

The eigenmodes of a system are characterized by both time parameter (frequency $-\omega$ ) and space propagation parameter (wave vector $-\vec{k}$ ). Then, the eigenmode is completely determined if the relation between the frequency and wave vector is known. This dispersion characteristic can be very simply determined for a homogeneous system. Indeed, in this system the Fourier transformation can be performed. In this case, the susceptibility of the system is dependent on $\omega$ and $\vec{k}$. As it is well known, the dispersion relations in this case can be determined by putting the pole part of susceptibility to zero $[9,10]$. This point has very simple explanation. Namely, let one defines the transmitting function of the system that connects the linear response of the system with the external (exciting) field - the effective susceptibility. In the case of homogeneous system, this connection can be presented as

$$
\vec{J}(\vec{k}, \omega)=\overrightarrow{\mathrm{X}}(\vec{k}, \omega) \vec{E}^{(e x t)}(\vec{k}, \omega),
$$

where $\vec{J}(\vec{k}, \omega)$ is the response of the system to external field $\vec{E}^{\text {ext }}(\vec{k}, \omega), \overrightarrow{\mathrm{X}}(\vec{k}, \omega)$ is the effective susceptibility.
The excitation of the eigenmodes means that action of infinitesimal field caused the nonzero response. It can be realized only if at $\vec{E}^{\text {ext }}(\vec{k}, \omega) \rightarrow 0, \overrightarrow{\mathrm{X}}(\vec{k}, \omega) \rightarrow \infty$. Because the effective susceptibility is connected with an initial susceptibility - the linear response on the total (local) field $\vec{\chi}(\vec{k}, \omega)$ - via self-energy part $\vec{S}(\vec{k}, \omega)$ as

$$
\overrightarrow{\mathrm{X}}(\vec{k}, \omega)=\left[\vec{\chi}^{-1}(\vec{k}, \omega)-\vec{S}(\vec{k}, \omega)\right]^{-1},
$$

then the condition of arising the eigenmodes can be written in the form

$$
\operatorname{det}\left[\vec{\chi}^{-1}(\vec{k}, \omega)-\vec{S}(\vec{k}, \omega)\right]=0
$$

Eq. (3) is the dispersion relation that gives us the set of the wave vectors and frequencies at which action of infinitesimal external field generates nonzero local currents.

In the case of inhomogeneous systems, the effective susceptibility can not be a simple function of the wave vector. The effective susceptibility will depend on the coordinate of a point in which the local current is determined. In other words, the problem is that the effective susceptibility is not the global characteristic of the system as it was in the homogeneous system case. The effective susceptibility here only describes the response of the point in the system on the external excitation. Obviously, this characteristic can not give us the information about general oscillating processes in the system. As an analog, the local current in a single element of an oscillating contour does not characterize the oscillating process in the whole contour.

Because the superconductor is characterized by a nonlocal response on the total field, the problem of determining the dispersion relation for a thin superconductor film falls into the kind of the problems mentioned above. The way that can lead to solution of the problem is in the finding the global characteristic of the system. That characteristic, obviously, can be the energy of external field absorbed by the system. It is clear that the resonance condition - or excitation of an eigenmodes leads to extremely strong absorption of energy of the external field by the system. The idea of calculation of the absorbed energy value to establish the eigenmode dispersion relations lies in the base of the present consideration.

\section{Linear response on the external field}

To solve of the problem of determining eigenmodes of the system under consideration, one needs to calculate the absorption of energy of external electromagnetic field acting on the system. For this purpose, one needs to introduce the linear response of the bulk superconductor on the local field $\vec{\chi}\left(\vec{R}, \vec{R}^{\prime} \omega\right)$. It is clear that this characteristic is the characteristic of the material. This linear response function connects the local currents inside the superconductor medium with the local electromagnetic field. In a general case of nonlocality, this connection has the form 
$\vec{J}(\vec{R}, \omega)=-i \omega \int_{V} d \vec{R}^{\prime} \ddot{\chi}\left(\vec{R}, \vec{R}^{\prime}, \omega\right) \vec{E}\left(\vec{R}^{\prime}, \omega\right)$,

where integration is performed over the particle volume. We shall represent the system under consideration as the superconductor film located in any medium. Then, for this system integration in Eq. (4) should be fulfilled over the film volume. For definiteness, we suppose that the medium consists of two semi-spaces with flat interface - the semiconductor substrate and external medium (Fig. 1).

The electrodynamical properties of the medium are characterized by the Green function $\vec{G}\left(\vec{r}, \vec{r}^{\prime}, \omega\right)$. As it is well known, for the considered medium, the Green function consists of two parts $[11,12]$

$\vec{J}(\vec{R}, \omega)=-i \omega \int_{V} d \vec{R}^{\prime} \vec{\chi}\left(\vec{R}, \vec{R}^{\prime}, \omega\right) \vec{E}\left(\vec{R}^{\prime}, \omega\right)$.

The first of them named as the direct part of the Green function describes the photon propagating in the upper medium. The second part of the Green function is named as indirect part. In far zone, it describes the reflection and transmission processes caused by the interface.

To calculate the energy of external field absorbed by the system under consideration, one needs to know the effective susceptibility that is the mean linear response on the external field. To perform this calculation one should consider the equation for self-consistent field. This equation is usually named as the Lippmann-Schwinger equation $[6,12]$ and for the system under consideration has the form

$$
\begin{aligned}
& E_{i}(\vec{k}, z, \omega)=E_{i}^{(e x t)}(\vec{k}, z, \omega)- \\
& -\frac{1}{\varepsilon_{0}} \frac{\omega^{2}}{c^{2}} \int_{0}^{h} d z^{\prime} G_{i j}\left(\vec{k}, z, z^{\prime}, \omega\right) \int_{0}^{h} d z^{\prime \prime} \chi_{j l}\left(\vec{k}, z^{\prime}, z^{\prime \prime}, \omega\right) E_{l}\left(\vec{k}, z^{\prime \prime}, \omega\right),
\end{aligned}
$$

with $\vec{k}$ wave vector in the plane of substrate surface, and $-i \omega \chi_{j l}\left(\vec{k}, z^{\prime}, z^{\prime \prime}, \omega\right)$ bulk nonlocal tensor of conductivity. In Eq. (6), the fact that the system in the plane of the interface is homogeneous was used for performing the Fourier transformation in the plane of the film (XOY plane, Fig. 1). Integration is made over the thickness of the film. The conductivity tensor connects the local currents with self-consistent local field

$J_{i}(\vec{k}, z, \omega)=-i \omega \int_{0}^{h} d z^{\prime \prime} \chi_{i l}\left(\vec{k}, z, z^{\prime \prime}, \omega\right) E_{l}\left(\vec{k}, z^{\prime \prime}, \omega\right)$.

The conductivity can be calculated in the microscopic approach. For example, this conductivity was calculated for BCS model of superconductivity in the frame of RPA in the work [13]. Using modified UV transformations method, the conductivity was calculated for superconductors with a strong coupling [14].

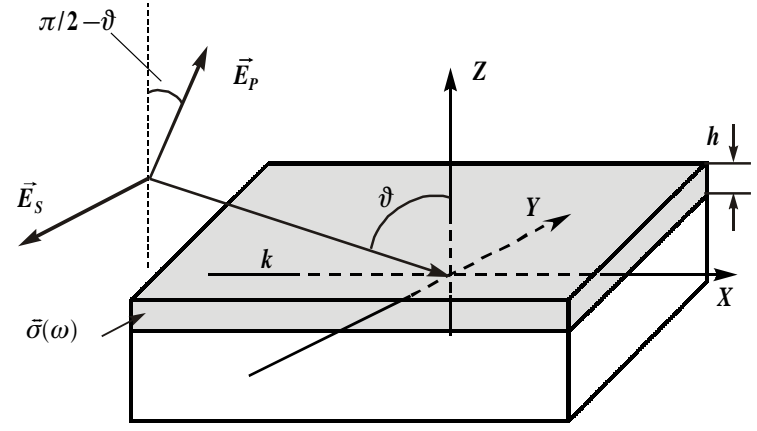

Fig. 1. Sketch of the system under consideration.

The analytical solution of Eq. (6) can be written in the terms of effective susceptibility $\chi_{i j}\left(\vec{k}, z, z^{\prime}, \omega\right)$ or linear response on external field that connects the local currents caused by external field with this external field

$$
J_{i}(\vec{k}, z, \omega)=-i \omega \int_{0}^{h} d z^{\prime \prime} \mathrm{X}_{i l}\left(\vec{k}, z, z^{\prime \prime}, \omega\right) E_{l}^{(e x t)}\left(\vec{k}, z^{\prime \prime}, \omega\right) .
$$

The solution can be obtained in the framework of the method developed in the works $[8,15]$ and has the form

$$
\mathrm{X}_{i j}\left(\vec{k}, z, z^{\prime}, \omega\right)=\chi_{i l}\left(\vec{k}, z, z^{\prime}, \omega\right) Q_{l j}\left(\vec{k}, z^{\prime}, \omega\right),
$$

with

$Q_{l j}\left(\vec{k}, z^{\prime}, \omega\right)=\left[\delta_{j l}+S_{j l}\left(\vec{k}, z^{\prime}, \omega\right)\right]^{-1}$.

The expression $S_{j l}\left(\vec{k}, z^{\prime}, \omega\right)$ has the meaning of selfenergy part and describes the corrections of a pole part of effective susceptibility by electrodynamical interactions in the system. For monochromatic external field, the self-energy part can be written in the form

$$
\begin{aligned}
& S_{j l}\left(\vec{k}, z^{\prime}, \omega\right)=\frac{\omega^{2}}{\varepsilon_{0} c^{2}} \int_{0}^{h} d z^{\prime \prime} G_{j k}\left(\vec{k}, z^{\prime}, z^{\prime \prime}, \omega\right) \times \\
& \times \int_{0}^{h} d z^{\prime \prime \prime} \chi_{k l}\left(\vec{k}, z^{\prime \prime}, z^{\prime \prime \prime}, \omega\right) \exp \left[i k_{Z}\left(z^{\prime}-z^{\prime \prime \prime}\right)\right],
\end{aligned}
$$

where $k_{z}$ is $z$-component of the wave vector of external field. If the external field is the plane wave with the incident angle $\vartheta$, then $k_{z}=(\omega / c) \cos \vartheta$.

Then, the linear response on the external field (the effective susceptibility) allows us to calculate the local fields and currents in the system. Namely, the self-consistent (local) field in arbitrary point in the $\vec{k},-z$ space can be calculated according to

$E_{i}(\vec{k}, z, \omega)=L_{i l}\left(\vec{k}, z, z^{\prime}, \omega\right) E_{l}^{(e x t)}\left(\vec{k}, z^{\prime}, \omega\right)$,

with the local-field operator 
$L_{i l}\left(\vec{k}, z, z^{\prime}, \omega\right)=\delta_{i l} \delta_{z, z^{\prime}}-$

$-\frac{1}{\varepsilon_{0}} \frac{\omega^{2}}{c^{2}} \int_{H} d z^{\prime} G_{i j}\left(\vec{k}, z, z^{\prime}, \omega\right) \int_{H} d z^{\prime \prime} \chi_{j l}\left(\vec{k}, z^{\prime}, z^{\prime \prime}, \omega\right) \ldots$.

One should note that representation of self-consistent field in the term of local-field operator instead of the standard representation via the local-field factor [12] caused by nonlocal nature of superconductivity requires to use the integral nonlocal constitutive equations [Eq. (7)]

\section{Absorption of external field energy}

Let us define the dissipative function as the energy of external electromagnetic field absorbed by the unit volume of the system for unit time. Then, normalized dissipative function can be written as

$\Omega(\omega, \vec{k})=\frac{\left\langle\overline{\left(\vec{J}+\vec{J}^{*}\right)\left(\vec{E}+\vec{E}^{*}\right)}>\right.}{I^{\text {ext }}}$,

where $\langle\ldots\rangle$ means the averaging over the volume and (...) over time, and $I^{\text {ext }}$ is the intensity of external field. Substituting Eqs. (8) and (12) into this equation and taking into account that $I^{\text {ext }}=E_{j}^{(e x t)}\left(E_{k}^{(e x t)}\right)^{*}$, one can obtain

$$
\begin{aligned}
& \Omega=-\frac{i \omega}{h} \int_{0}^{h} d z \int_{0}^{h} d z^{\prime}\left[\mathrm{X}_{i j}\left(z, z^{\prime}, \omega\right) L_{i k}^{*}(z, \omega) e^{i k_{Z}\left(z-z^{\prime}\right)}-\right. \\
& \left.-\mathrm{X}_{i k}^{*}\left(z, z^{\prime}, \omega\right) L_{i j}(z, \omega) e^{-i k_{Z}\left(z-z^{\prime}\right)}\right] \tau_{j} \tau_{k}
\end{aligned}
$$

where $\tau_{i}$ is the unit vector of the external field polarization.

It should be noted that in the case of a local constitutive equation (London-type superconductor)

$$
J_{i}(\vec{k}, z, \omega)=-i \omega \chi_{i l}(\omega) E_{l}(\vec{k}, z, \omega)
$$

the connection between the current and external field has the form

$$
J_{i}(\vec{k}, z, \omega)=-i \omega X_{i l}^{(l o c)}(\vec{k}, z, \omega) E_{l}^{(e x t)}(\vec{k}, z, \omega),
$$

where

$$
\mathrm{X}_{i l}^{(l o c)}(\vec{k}, z, \omega)=\chi_{i k}(\omega) Q_{k l}^{(L)}(\vec{k}, z, \omega),
$$

and

$$
\begin{aligned}
& Q_{k l}^{(L)}(\vec{k}, z, \omega)= \\
& =\left[\delta_{l k}+\frac{\omega^{2}}{\varepsilon_{0} c^{2}} \int_{0}^{h} d z^{\prime} G_{l m}\left(\vec{k}, z, z^{\prime}, \omega\right) \chi_{m l}(\omega) e^{i k_{z}\left(z-z^{\prime}\right)}\right]^{-1}
\end{aligned}
$$

Using Eqs.(15) and (16) one obtains the connection between local and external fields
$E_{j}(\vec{k}, z, \omega)=\left(\chi_{i j}(\omega)\right)^{-1} X_{i l}(\vec{k}, z, \omega) E_{l}^{(e x t)}(\vec{k}, z, \omega)$.

Then, the dissipative function of the film of Londontype superconductor is

$$
\begin{aligned}
& \Omega=-i \omega \frac{1}{h} \int_{0}^{h} d z\left[\mathrm{X}_{i j}(\vec{k}, z, \omega) \mathrm{X}_{j k}{ }^{*}(\vec{k}, z, \omega)\left(\chi_{i k}{ }^{*}(\omega)\right)^{-1}-\right. \\
& \left.-\mathrm{X}_{j i}{ }^{*}(\vec{k}, z, \omega)\left(\chi_{k i}(\omega)\right)^{-1} \mathrm{X}_{k j}(\vec{k}, z, \omega)\right] \times \\
& \times E_{j}^{(e x t)}(\vec{k}, z, \omega)\left(E_{j}^{(e x t)}(\vec{k}, z, \omega)\right)^{*} .
\end{aligned}
$$

Taking into account Eq. (17) one obtains

$$
\begin{aligned}
& \Omega(\vec{k}, \omega)=2 \omega \frac{1}{h} \int_{0}^{h} d z \operatorname{Im}\left(\chi_{i j}(\omega)\right) Q_{i k}^{(L)}(\vec{k}, z, \omega) \times \\
& \times\left(Q_{j l}^{(L)}(\vec{k}, z, \omega)\right)^{*} \tau_{k} \tau_{l} .
\end{aligned}
$$

Because electrical susceptibility for superconductor is a real value, Eq. (21) shows us that superconductor film of London-type can not absorb energy of external field. This, at first view, strange result has very simple explanation. Namely, for the nano-scale objects, the local field is a very rapidly varying value. Then, all the interactions inside the object are nonlocal. In this connection, one can assert that only nonlocal models for superconductivity one has to consider for studying electrodynamical interactions in the system containing the small superconducting particles (or thin films). To analyze the dispersion relations of the small object, one should to attentively consider the experimental set up to excite any eigenmode in the bounded system. Let us, for example, consider the experimental set up for surface wave excitations. The most widely used method of excitation of surface plasmon-polariton is the method of attenuated internal reflectivity (Fig. 2) in which the probing beam radiates the film placed at the surface of ATR-prism. The intensity of reflected radiation is measured as a function of the incidence angle $\vartheta$. The excitation of the surface wave that occurs at the angle $\vartheta_{R}$ is characterized by abrupt decrease of the reflection signal at the detector. This surface plasmon-polariton resonance is characterized by strong absorption of the external field energy by

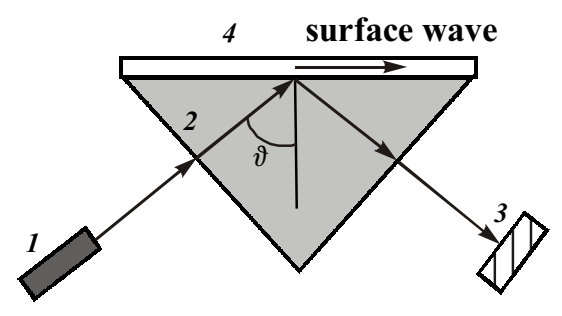

Fig. 2. Set up for excitation of surface wave by the attenuated total internal reflectivity (ATR) method. 1 - light source (laser); 2 -ATR prism; 3 - detector; 4 - the film in which the surface wave is excited. 
the system under consideration. It means that the dissipative function has a maximum at any values of frequency $\omega$ and wave vector $k=\sqrt{\varepsilon_{\text {prism }}}(\omega / c) \sin \vartheta_{R}$. It is obvious that the set of pairs frequency-wave vector composite the curves that are the dispersion relations of eigenmodes. To demonstrate this, method we shall calculate the absorption of the external electrodynamic field energy for thin superconducting film placed at the surface of semiconductor.

\section{Numerical calculations and discussion}

To calculate the energy absorbed by the film, one needs to know the effective susceptibility and local-field operator [Eqs. (9) and (14)]. From the other side, to calculate these values, one should know the linear response to the local field $\chi_{i j}\left(\vec{R}, \vec{R}^{\prime}, \omega\right)$. One should perform a calculation of the function $\chi_{i j}\left(\vec{R}, \vec{R}^{\prime}, \omega\right)$ in the frame of any microscopic model. There are a big number of models (see, for example, Refs [17-21]) in the frame of which the numerous attempts to describe the HTSC phenomena were
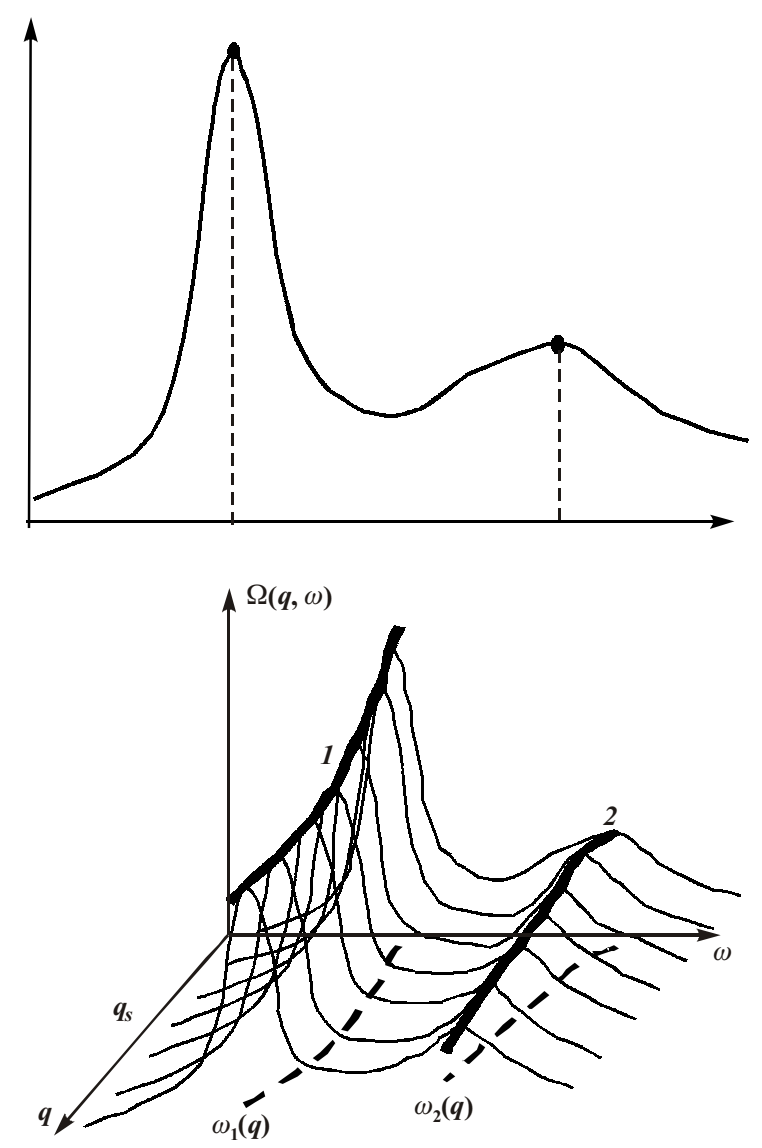

Fig. 3. The method of dispersion relations determination. The profile of the dissipative function in the cross-section $q=q_{s}$ (top). The dissipative function image in $\Omega, q, \omega$ - space (bottom). The projection of the local maxima points on the $q, \omega$-plane are the functions $\omega_{1}(q)$ and $\omega_{2}(q)$ play the role of two branches of dispersion relations of eigenmodes of the system. performed. Despite this fact, most of the properties of HTSC materials can be rather adequately described using the phonon model with strong coupling. Then, for simplicity, we shall use this model $[22,23]$. Here, we shall use the approach proposed in [16] that is based on the modified $u-v$ transformations. The linear response to the local field $\chi_{i j}\left(\vec{R}, \vec{R}^{\prime}, \omega\right)$ for strong-coupling superconductor was calculated in the framework of RPA using modified $u-v$ transformation method [16]. Using this nonlocal linear response function, one can calculate the energy of external field absorbed by superconducting film at the semiconductor substrate.

Then, we shall assume that thickness of the superconductor film is much less then the wavelength of the external field. At the same time, to use mesoscopic approach to describe the electrodynamical properties of the system, one needs to suppose that film thickness is much larger than the lattice constant or $h>>1 / k_{D}\left(k_{D}\right.$ is the Debye wave vector). Moreover, the thickness $h$ should be comparable to the Pippard penetration length $\Lambda_{P}$. This requirement provides the penetration of the field into the whole thickness of the film. These requirements are rather good satisfied for HTSC, where the values of energy gap are rather great $\left(\omega_{g}=\Delta / \hbar \approx 10^{13} \div 10^{15} \mathrm{~Hz}\right)$ and the Cooper pair dimensions are about few nanometers. Then the films with the thickness $h \approx 10^{-7} \div 10^{-9} \mathrm{~m}$ are satisfy the abovementioned conditions.

In the frame of random phase approximation (RPA), the linear response function can be written in the form [13]

$$
\vec{\chi}(\vec{k}, \omega)=\frac{n e^{2}}{m}\left[\vec{U}+\alpha(\omega)\left(\frac{k}{\omega}\right)^{2}\left(\vec{U}+\frac{2 \vec{k} \otimes \vec{k}}{k^{2}}\right)\right],
$$

where $m$ and $e$ are the mass and charge of the charge carriers, respectively. Symbol $\otimes$ means the direct tensor product. $\vec{U}$ is the unit tensor. The calculations were made in the frame of the so-called method of the pseudo-vacuum Green function [12]. The main idea of the method consists in consideration of the object (here it is the film) situated in any medium (pseudo-vacuum), electrodynamical properties of which are supposed as known. In the case under consideration, this medium consists of two semi-spaces. Upper semi-space is vacuum and lower semispace is semiconductor substrate characterized by dielectric function $\varepsilon_{1}(\omega)$. For definiteness, we suppose that the dielectric function has the fallowing form :

$\varepsilon_{1}(\omega)=\varepsilon_{\infty} \frac{\omega_{L O}{ }^{2}-\omega_{T O}^{2}}{\omega_{T O}^{2}-\omega^{2}-i \omega \gamma}$

where $\varepsilon_{\infty}$ is the high frequency dielectric constant; $\omega_{L O}$ and $\omega_{T O}$ are the frequencies of longitudinal and transverse optical phonons, respectively; $\gamma$ is decay constant.

As it was mentioned above, the electrodynamical Green function of the medium in which the film is situated (two semi-spaces with flat interface) consists of two parts and has the form [Eq. (5)]. Because the system un- 


\section{Lozovski, D. Reznik: Electrodynamic linear response of a superconductor film ...}

der consideration is homogeneous in the plane of interface, it is convenient to use the so-called $\vec{k}-, z-$ representation. In this representation, direct and indirect parts of the Green function in the coordinate system where $\vec{k}=\left(k_{x}=k, k_{y}=0\right)$ can be written in the form $\left(k=k_{0} \sin \vartheta\right)$

$\vec{D}\left(\vec{k}, z-z^{\prime}, \omega\right)=\vec{g}\left(\vec{k}, z-z^{\prime}, \omega\right) e^{i \eta_{0}\left|z-z^{\prime}\right|}$

$-\frac{\vec{u}_{z} \otimes \vec{u}_{z}}{k_{0}^{2}} \delta\left(z-z^{\prime}\right)$,

and

$$
\begin{aligned}
& I_{i j}\left(k, z+z^{\prime}, \omega\right)= \\
& =2 \pi i e^{i \eta_{0}\left(z+z^{\prime}\right)}\left(\begin{array}{ccc}
\eta_{0} R_{p} & 0 & k \cdot R_{p} \\
0 & \left(k_{0}^{2} / \eta_{0}\right) R_{s} & 0 \\
-k \cdot R_{p} & 0 & -\left(k^{2} / \eta_{0}\right) R_{p}
\end{array}\right) .
\end{aligned}
$$

In these equations, the next designations are used:
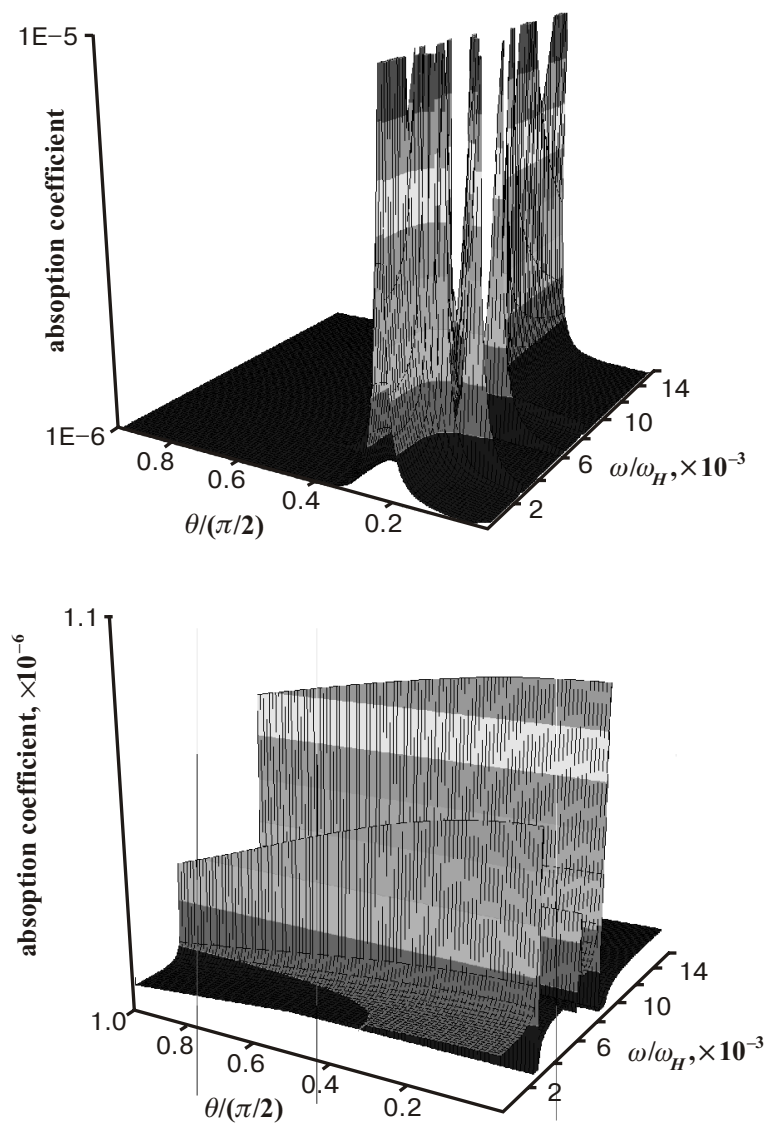

Fig. 4. The view of the normalized dissipative function of superconducting film at a semiconductor substrate for different polarizations of external field. The top pannel corresponds to absorption of $p$-polarized wave. The bottom panel corresponds to absorption of $s$-polarized wave.

$$
\begin{aligned}
& \vec{g}\left(\vec{k}, z-z^{\prime}, \omega\right)= \\
& =\frac{i}{2 k_{0}^{2}}\left(\begin{array}{ccc}
\eta_{0} & 0 & -k \cdot \operatorname{sign}\left(z-z^{\prime}\right) \\
0 & k_{0}^{2} / \eta_{0} & 0 \\
-k \cdot \operatorname{sign}\left(z-z^{\prime}\right) & 0 & k^{2} / \eta_{0}
\end{array}\right),
\end{aligned}
$$

and $\eta=\sqrt{\varepsilon k_{0}^{2}-k^{2}}, \eta_{0}=\sqrt{k_{0}^{2}-k^{2}}$.

Moreover, $R_{p}=\frac{\eta-\varepsilon \eta_{0}}{\eta+\varepsilon \eta_{0}}, R_{s}=\frac{\eta_{0}-\eta}{\eta_{0}+\eta}$ are the Fresnel coefficients for reflection of $p$ - and $s$ - polarized waves, respectively.

The results of numerical calculations are shown in Figs. 4 and 5. In Fig. 5, the projections of the ridges of dissipative function "mountains" are shown. The lines of these projections are the dispersion relations of electromagnetic waves localized at the superconductor film. We can see that three branches of plasmon oscillations caused by the complicated structure of the dispersion law of su-
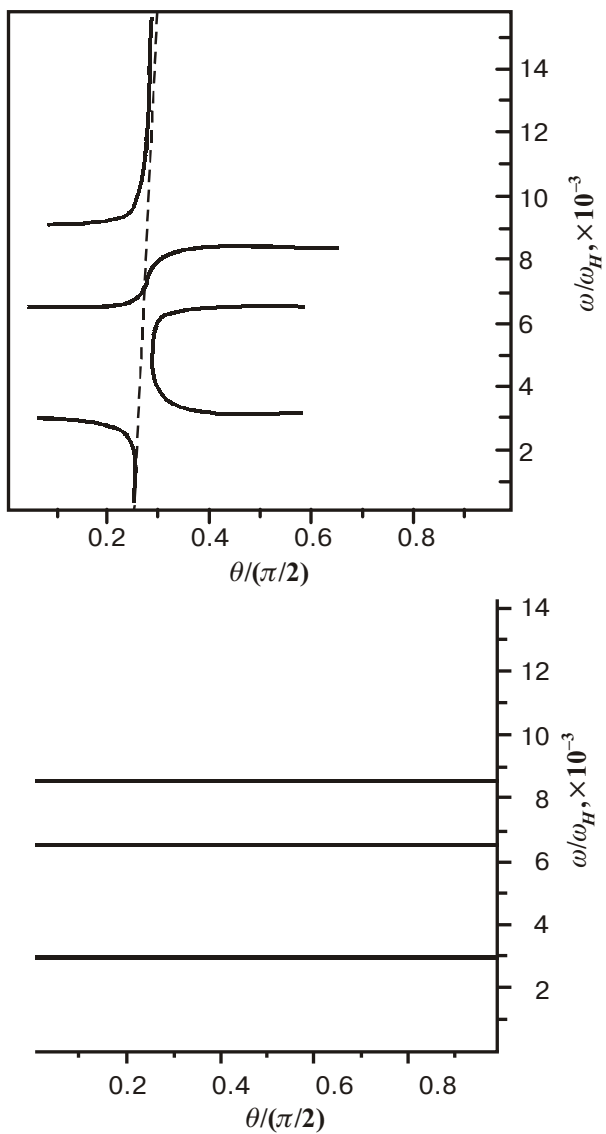

Fig. 5. The dispersion curves of the waves $p$-polarized (top) and $s$-polarized (bottom) localized at the superconducting films, which are the eigenmodes of the system. 


\section{Lozovski, D. Reznik: Electrodynamic linear response of a superconductor film ...}

perconductor which are only slightly renormalized by electromagnetic interactions in the case of excitation of $s$-polarized waves, in the case of $p$-polarized eigenmodes change very strongly. Namely, the interaction with the external field (its dispersion law is shown by the dashed line) of the plasmon oscillations results in to splitting and occurring the energy gaps in the dispersion relations of eigenmodes. One should to note that the question of stability of these excitations should be considered in a special study.

\section{References}

1. M. Suzuki, Hall coefficients and optical properties of $\mathrm{La}_{2-\mathrm{x}} \mathrm{Sr}_{\mathrm{x}} \mathrm{CuO}_{4}$ single-crystal thin films // Phys. Rev. B 39, pp. 2312-2321 (1989).

2. Guo-chun Liang, Xiaohong Dai, David F. Hebert, Theodore Van Duzer, Nathan Newman, and Brady F.Cole, High-temperature superconductor resonators and phase shifters // IEEE Transactions on Appl. Superconductivity, 1, pp. 58-66 (1991).

3. J.-Ho Choy, S.-J. Kwon, S.-J. Hwang, Y.-I. Kim, and W. Lee, Interaction route to nano-hybrids: inorganic/organichigh TC cuprate hybrid materials // J. Of Mat.Chem., 8, pp. 129-135 (1999).

4. I. Iguchi, E. Kume, and H. Takahashi, Emitted spectra of electromagnetic waves from a tunel-injected nonequilibrium hogh-TC $\mathrm{YBa}_{2} \mathrm{Cu}_{3} \mathrm{O}_{7-\mathrm{y}}$ superconductor // Phys.Rev., B62, pp. 5370-5373 (2000).

5. K. Halterman and O. T.Valls, Nonlinear electrodynamics of p-wave superconductors // Phys. Rev., B62, pp. 5904-5912 (2000).

6. J.-J. Greffet and R. Carminati, Image formation in near-filed optics // Prog. Rep. Sci., 56, pp. 133-237 (1997).

7. B.I. Lembrikov, M.A. Itskovsky, H. Cohen, and T. Maniv, Electrodynamics in the near-field regions of anisotropic nanoscopic films and platelets // Phys. Rev., B67, 085401 (2003).

8. S. Bozhevolnyi and V. Lozovski, Self-consistent model for second-harmonic near-field microscopy // Phys. Rev., B61, pp. 11139-11150 (2000).
9. A.A. Abrikosov, L.P. Gor'kov and I.Ye. Dzyaloshinskii, Quantum Field Theoretical Methods in Statistical Physics (Pergamon Press, Oxford, 1965).

10. E.M. Lifshits and L.P. Pitaevskii, Statistical Physics Part 2, (Course of Theoretical Physics: 9) (Pergamon Press, Oxford, 1980).

11. P.M. Morse and H. Feshbach, Methods of Theoretical Physics Part 1 (McGraw-Hill Book Company Inc., New York, 1953).

12. O. Keller, Local fields in the electrodynamics of mesoscopic media // Phys. Rep., 268, pp. 85-262 (1996)

13. O. Keller, Electromagnetic surface waves on a Cooper-paired superconductor // J. Opt. Soc. Am., B7, pp. 2229-2235 (1990).

14. M.V. Glumova, V.Z. Lozovski and D.V. Reznik, Surface waves on a superconductor: beyond the weak-coupling approximation // J. Phys.: Condens. Matter, 14, pp. 4271-4285 (2002).

15. V. Lozovski, Yu. Nazarok and S. Bozhevolnyi, Near-field imaging of pyramid-like nanoparticles at a surface // Physica, E11, pp. 323-331 (2001)

16. B. Khudik, V. Lozovski, and A. Nazarenko, Theory of superconductors with strong coupling in the Bogolyubov method // Physica Status Solidi (b) 148, pp. 297-303 (1988).

17. D.F. Mattis, Electron-phonon interaction in two dimensions: The case for strong coupling in High-TC superconductors // Phys. Rev., B36, pp. 3933-3936 (1987).

18. E. Pashytskii, About mechamism of high temperature superconductivity in $\mathrm{YBa}_{2} \mathrm{Cu}_{3} \mathrm{O}_{7}^{-} / /$Fiz. Tverd. Tela (Sov. Solid State Phys.), 31, pp. 46-56 (1989).

19. I.Ye. Dziyaloshinskii, About chemical nature of hole coupling in high temperature superconductors // Pisma v ZhETF, 49, pp. 119-121 (1989).

20. C. Rao, A. Ganguli, Structural aspects of superconducting cuprates // Acta Crystallographica, 51, pp. 604-720 (1995).

21. V. Moschalkov, J. Vanacken, and L. Trappeniers, Phase diagram of high-TC cuprates: Stripes, pseudogap, and effective dimensionality // Phys.Rev., B64, p. 214504 (2001).

22. G.M. Eliashberg, Interaction of electrons with the lattice oscillations in superconductor // ZhETPh, 38, pp. 966-976 (1960).

23. D.J. Scalapino, J.R. Schrieffer, J.W. Wilkins, Strong-coupling superconductivity // Phys.Rev. 148, pp. 263-279 (1966).

24. T.R. Yang, M.M. Dvoynenko, A.V. Goncharenko and V.Z. Lozovski, An exact solution of the Lippmann-Schwinger equation in one dimension // Am. J. Phys., 71, pp. 64-71 (2003). 